\title{
THE RATIONALE FOR USING LOGARITHMIC TRANSFORMATION OF CONCENTRATION DATA IN TOXICOKINETIC STUDIES
}

\author{
Toshiji IGARASHI \\ Department of $R \& D$ Information Management, Eisai Co., Ltd., \\ Koishikawa 4-6-10, Tokyo 113, Japan
}

Received October 17, 1994 ; Accepted November 24, 1994

\begin{abstract}
The present paper focuses on the necessity of logarithmic transformation of plasma concentration in toxicokinetic studies. The dose-plasma concentration relationship is the most important subject to be studied, because test doses rise over a wide range from therapeutic dose level to toxic dose level in toxicity studies. It is concluded that the logarithmic transformation of concentration data effectively stabilized the variances, and the regression analysis of log-transformed data makes it possible to quantitatively evaluate the magnitude of non-linear kinetics of test compounds in toxicity studies.
\end{abstract}

KEY WORDS : Logarithmic transformation, Toxicokinetics, Statistical analysis, Homogeneity of variances, Dose-concentration relationship.

\section{INTRODUCTION}

The primary objective of toxicokinetics is to describe the systemic exposure to the test compounds achieved in the test animals (FDA Notice, 1994). The dose-concentration relationship, change in plasma concentration during the course of repeated dose toxicity studies and sex differences should be examined. The analysis of variance (ANOVA) may be the most practical and robust method for the statistical analysis of those factorial effects and their interactions. Among the subjects to be analyzed, the dose-plasma concentration relationship is the most important because it validates the toxicity study and provides data useful for interpreting

The contents of this paper were published in Drug Information Journal, Vol. 28, pp. 191-194, 1994, in a form of short communication.

Correspondence : Toshiji IGARASHI at the above address. results of the study. The regression analysis of dose-dependency in toxicokinetic studies should focus on the fact that there are variable cases of non-linear kinetics; the plasma concentration does not increase in a dose-dependent manner because of saturation of the absorption process in some cases, but it increases super-proportionally with increasing dose due to saturation and change in metabolism and excretion in other cases.

Assumption of normality of distribution and homogeneity of variance are required for the test of statistical significance of differences in mean values between or among groups by $t$-test or F-test. Concerning this assumption, a recent issue of WHO draft guideline for testing the equivalence of generic products recommends that the plasma concentration and concentrationrelated quantities should preferably be analyzed after logarithmic transformation (WHO, 1993). Although there are some papers indicating the necessity of logarithmic data-transformation in bioequivalence studies (Westlake, 1988 ; Midha et al., 1993), there are, to my knowledge, no paper 
concerning this issue in the field of toxicokinetic studies. The logarithmic transformation of the concentration data seems to be especially important for the statistical analysis of the data in toxicokinetic studies, because test doses rise over a wide range from therapeutic dose level to toxic dose level in toxicity studies, and the concentration data such as area under concentration-time curve (AUC), maximum plasma concentration $\left(\mathrm{C}_{\max }\right)$ and plasma concentration at specified time after administration of a given dose $\left(\mathrm{C}_{\text {time }}\right)$, usually vary over an extremely wide range.

For the purpose to investigate the rationale for using logarithmic transformation of plasma concentration, a small set of plasma concentration data obtained in an actual toxicity study was statistically analyzed with and without logarithmic data-transformation.

\section{MATERIALS AND METHODS}

A small set of plasma concentration data is shown in Table 1. To make story short and simple, the data referred to here were limited to only $\mathrm{C}_{\max }(\mu \mathrm{g} / \mathrm{ml})$ determined at the end of 12 month repeated dose toxicity study. Test dose levels were $0.2,0.7$ and $2.0 \mathrm{mg} / \mathrm{kg}$, and four male and four female dogs were allocated to each test dose level. It is doubtful what conclusions we can draw from this simple list of individual data (24 data in total) without statistical analysis. For example, are there any sex difference in the plasma concentration? What kind of doseconcentration relationship can we find in these data? We need some appropriate methods for the statistical analysis to draw conclusions from some a complicated set of actual toxicokinetic data.

ANOVA may be the most practical and robust method for the statistical analysis of factorial effects such as sex of test animals, test dose levels and time-course of repeated dosing and interactions among those factors. The regression analysis is a useful tool for the examination of dose-dependency in toxicokinetic studies. Test dose levels in the present case study, 0.2, 0.7 and $2.0 \mathrm{mg} / \mathrm{kg}$, were regarded as nearly equidistant when they were plotted on a logarithmic scale, and the regression analysis was conducted by using an orthogonal polynominal.

\section{RESULTS AND DISCUSSION}

Analysis of variance of data with and without data transformation: Results of the analysis of variance of raw (untransformed) data and logtransformed data are comparatively shown in Table 2. As shown in ANOVA 1 (untransformed data), both factorial effects of sex and dose and the interaction of sex and dose were all statistically significant. However, in ANOVA 2 (log-transformed data), sex and dose were significant but the interaction of sex and dose was not statistically significant.

Variances of data within respective male and female groups at each test dose level are shown in Table 3. When data were not transformed, variances within respective groups varied an extremely wide range from 0.0033 to 2.0590 , while variances of log-transformed data of male and female groups were in a comparable range from 0.017 to 0.070 . In brief, the variance of raw data was heteroscedastic but the logarithmic transformation made it homoscedastic.

When the concentration data were not transformed, the larger the mean value was, the larger the standard deviation (SD) was. As indicated in Table 4, the coefficient of variances (SD/mean) were all comparable with each other $(0.32-0.50)$, indicating that $\mathrm{SD}$ values were proportional to their mean values. It has been generally known that the logarithmic transformation of data effectively stabilized the variances, when SD values

Table 1. Plasma concentration $\left(\mathrm{C}_{\max }, \mu \mathrm{g} / \mathrm{ml}\right)$ obtained at the end of a 12 month repeated dose toxicity study in dogs ( $\mathrm{n}$; number of animals per group studied).

\begin{tabular}{ccccccccc}
\hline Dose & \multicolumn{4}{c}{ Male $(\mathrm{n}=4)$} & \multicolumn{5}{c}{ Female $(\mathrm{n}=4)$} \\
\hline $0.2 \mathrm{mg} / \mathrm{kg}$ & 0.143 & 0.089 & 0.229 & 0.160 & 0.087 & 0.153 & 0.267 & 0.311 \\
$0.7 \mathrm{mg} / \mathrm{kg}$ & 0.506 & 0.330 & 0.555 & 0.939 & 0.746 & 0.416 & 0.703 & 1.009 \\
$2.0 \mathrm{mg} / \mathrm{kg}$ & 1.300 & 1.001 & 1.084 & 1.960 & 3.697 & 4.473 & 1.184 & 3.713 \\
\hline
\end{tabular}


Table 2. Comparison of ANOVAs of untransformed and log-transformed data :

ANOVA 1: Raw data.

\begin{tabular}{lrrrr}
\multicolumn{1}{c}{ Source } & DF & \multicolumn{1}{c}{ SS } & MS & \multicolumn{1}{c}{ F } \\
\hline Sex & 1 & 2.9843 & 2.9843 & $7.50^{*}$ \\
Dose & 2 & 19.8629 & 9.9314 & $24.97^{* *}$ \\
Sex $\times$ Dose & 2 & 4.5112 & 2.2556 & $5.67^{*}$ \\
Residual & 18 & 7.1596 & 0.3978 & \\
\cline { 1 - 4 } Total & 23 & 34.5178 & & \\
\hline
\end{tabular}

* and " indicate the significance at $\mathrm{P}=0.05$ and 0.01 , respectively. ANOVA 2 : Log-transformed data.

\begin{tabular}{lrccc}
\hline \multicolumn{1}{c}{ Source } & DF & SS & MS & F \\
\hline Sex & 1 & 0.2013 & 0.2013 & $5.03^{*}$ \\
Dose & 2 & 4.6191 & 2.3096 & $57.60^{* *}$ \\
Sex $\times$ Dose & 2 & 0.0886 & 0.0443 & 1.10 \\
Residual & 18 & 0.7177 & 0.0399 & \\
Total & 23 & 5.6267 & & \\
\hline
\end{tabular}

$*$ and $*$ indicate the significance at $\mathrm{P}=0.05$ and 0.01 , respectively.

Table 3. Comparison of variance of data with and without data transformation : Variances of data within respective male and female groups consisting of 4 animals per group at each test dose level are shown.

\begin{tabular}{cccccc}
\hline & \multicolumn{2}{c}{ Raw data } & \multicolumn{2}{c}{ Log-transformed data } \\
\cline { 3 - 4 } Dose & Male & Female & & Male & Female \\
\hline $0.2 \mathrm{mg} / \mathrm{kg}$ & 0.0033 & 0.0106 & 0.0287 & 0.0635 \\
$0.7 \mathrm{mg} / \mathrm{kg}$ & 0.0658 & 0.0590 & 0.0348 & 0.0256 \\
$2.0 \mathrm{mg} / \mathrm{kg}$ & 0.1888 & 2.0590 & 0.0170 & 0.0698 \\
\hline
\end{tabular}

Table 4. Mean values and standard deviations (SD) for male and female groups at each test dose level (raw data).

\begin{tabular}{|c|c|c|c|c|c|c|}
\hline \multirow[b]{2}{*}{ Dose } & \multicolumn{3}{|c|}{ Male $(n=4)$} & \multicolumn{3}{|c|}{ Female $(n=4)$} \\
\hline & Mean & $\mathrm{SD}$ & $\mathrm{CV}^{*}$ & Mean & $\mathrm{SD}$ & $\mathrm{CV}^{*}$ \\
\hline $0.2 \mathrm{mg} / \mathrm{kg}$ & 0.155 & 0.058 & 0.37 & 0.205 & 0.103 & 0.50 \\
\hline $0.7 \mathrm{mg} / \mathrm{kg}$ & 0.583 & 0.257 & 0.47 & 0.719 & 0.243 & 0.34 \\
\hline $2.0 \mathrm{mg} / \mathrm{kg}$ & 1.336 & 0.435 & 0.32 & 3.267 & 1.435 & 0.44 \\
\hline
\end{tabular}

are proportional to their mean values.

Whether or not a set of plasma concentration data forms a normal distribution can not be discussed in this paper because of the small number of data. However, it is generally known that untransformed data of plasma concentrations do not verify the normality because the distribution is usually deformed by existence of some extraordinary large figures. This may be the reason why median values are recommended to represent a group of plasma concentration data. Arithmetic mean values estimated from untransformed data are usually greater than the median values and geometric mean values (unpublished data). Table 5 compares the arithmetic and geometric mean values and median values of raw data in Table 1. All mean values estimated from raw data (arithmetic mean values) were more or less greater than those calculated from the logtransformed data (geometric mean values). The use of median values for groups of small size like this case study seems to be irrelevant, and a non-parametric analysis of concentration data causes a great loss of information to be available from a toxicokinetic study.

Regression analysis of transformed data: $\mathrm{Fi}$ gure 1 comparatively shows dose-concentration relationship for untransformed data and logtransformed data. As indicated by ANOVA 1 (raw data), the interaction of sex and dose was statistically significant, suggesting in this case that the slope of dose-concentration curve (regression coefficient ) in female animals was steeper than that in male animals, or in other words, that there was no sex difference in plasma concentration at the low dose level but plasma concentration in female animals was much higher than male animals when they were administered higher doses. However, because of violation of the assumption of equality of variances and normality for each group, results of regression analysis of untransformed data may not be much informative.

On the other hand, as indicated by ANOVA 2 (log-transformed data), there was no interaction of sex and dose, suggesting that the plasma concentration was higher in female animals than that in male animals throughout three tested dose levels, and that the pattern (or slope) of doseconcentration relationship was the same for both male and female animals. Because there was 
Table 5. Comparison of arithmetic mean, geometric mean and median (raw data) for male and female groups at each test dose level.

\begin{tabular}{cccccccc}
\hline & \multicolumn{3}{c}{ Male $(\mathrm{n}=4)$} & & \multicolumn{3}{c}{ Female $(\mathrm{n}=4)$} \\
\cline { 2 - 3 } \cline { 7 - 8 } Dose & Arithmetic & Geometric & Median & & Arithmetic & Geometric & Median \\
\hline $0.2 \mathrm{mg} / \mathrm{kg}$ & 0.155 & 0.147 & 0.152 & & 0.205 & 0.182 & 0.210 \\
$0.7 \mathrm{mg} / \mathrm{kg}$ & 0.583 & 0.543 & 0.531 & & 0.719 & 0.685 & 0.725 \\
$2.0 \mathrm{mg} / \mathrm{kg}$ & 1.336 & 1.289 & 1.192 & & 3.267 & 2.920 & 3.705 \\
\hline
\end{tabular}
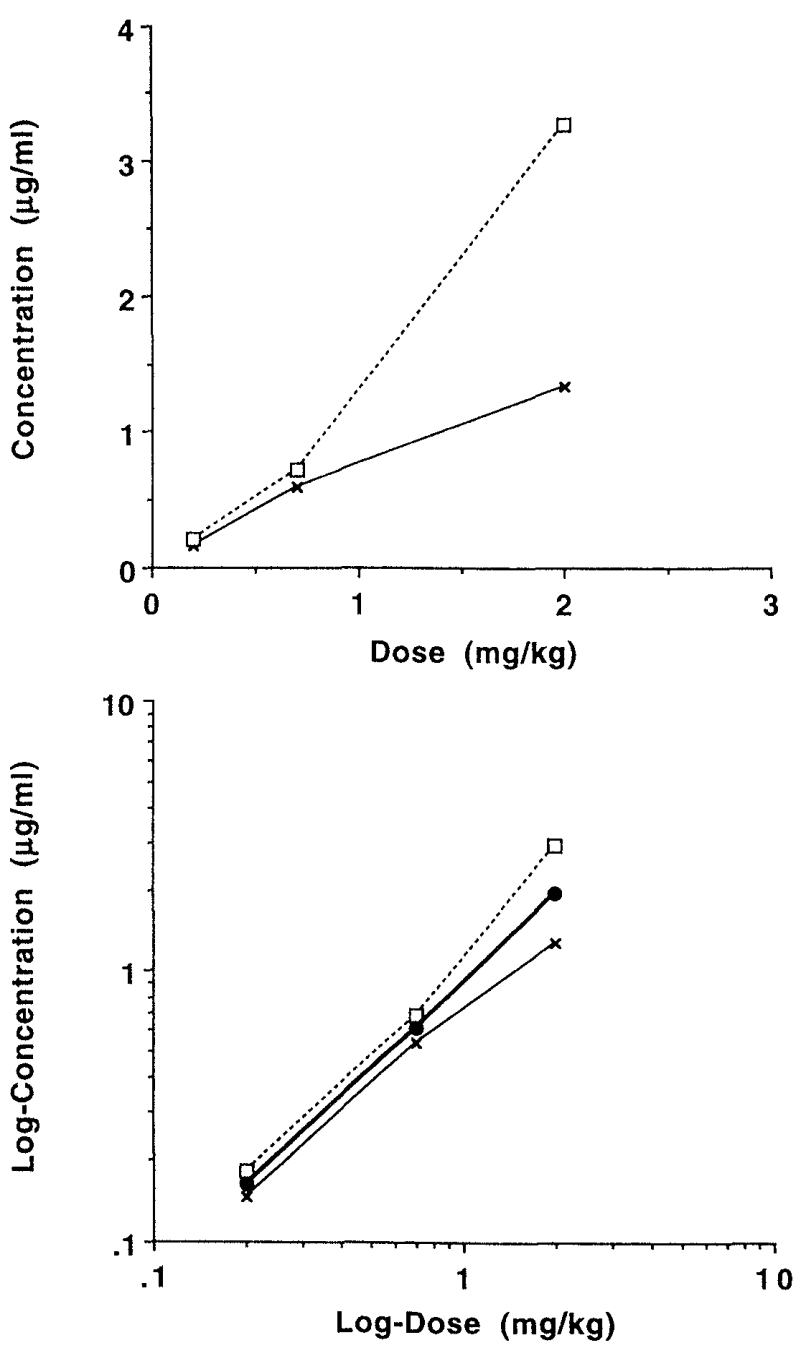

Fig. 1. Dose-concentration curve : Arithmetic mean values are plotted on linear scale (upper) and geometric mean values are on logarithmic scale (lower).

$-x-$ : male animals $(n=4), \cdots \square \cdots$ : female animals $(n=4),-$ : pooled data of both sexes $(n$ $=8$ )
Table 6. ANOVA 3 : Regression analysis of logtransformed data.

\begin{tabular}{|c|c|c|c|c|}
\hline Source & DF & SS & MS & $\mathrm{F}$ \\
\hline Sex & 1 & 0.2013 & & $5.03^{*}$ \\
\hline Dose & 2 & 4.6191 & - & - \\
\hline Linear & 1 & 4.6128 & 4.6128 & $115.61^{* *}$ \\
\hline Deviation $^{(}$ & 1 & 0.0063 & 0.0063 & 0.16 \\
\hline Sex $\times$ Dose & 2 & 0.0886 & 0.0443 & 1.10 \\
\hline Residual & 18 & 0.7177 & 0.0399 & \\
\hline Total & 23 & 5.6267 & & \\
\hline
\end{tabular}

${ }^{*}$ and ${ }^{* *}$ indicate the significance at $\mathrm{P}=0.05$ and 0.01 , respectively.

(a) : Deviations from linearity.

recognized no interaction of sex and dose, the variance and coefficient of linear regression were calculated from pooled data of both sexes $(n=8)$, as shown in Table 6. The factorial effects of dose $(D F=2)$ were decomposed to the linear regression $(\mathrm{DF}=1)$ and the deviation from linearity $(\mathrm{DF}=1)$. The variance for linear regression was highly significant but the variance for deviations from linearity was negligible.

The estimated coefficient of linear regression (slope) was 1.07. The estimated regression curves were :

$\mathrm{Y}=-0.132+1.07 \mathrm{X}$ for male animals, and $\mathrm{Y}=0.050+1.07 \mathrm{X}$ for female animals where $\mathrm{Y}$ is log-transformed concentration (y) and $\mathrm{X}$ is log-transformed dose level (x).

The intercepts, $\mathrm{a}=-0.132$ and 0.050 for male and female animals, are $\mathrm{Y}$ values at the dose $\mathrm{X}=0.0 \quad(\mathrm{x}=1.0 \mathrm{mg} / \mathrm{kg})$, i.e. $\mathrm{y}=0.738$ and $1.122 \mu \mathrm{g} / \mathrm{ml}$ for male and female animals. The sex difference in these values remained to be clarified. Although the possible sex difference in plasma concentration levels due to sex differ- 
ence in metabolic enzyme activities in rats is generally known, sex differences in distribution (e.g. due to definite differences in body size and form between male and female animals) and other factors should be taken into consideration.

$A$ proposal for quantitative evaluation of nonlinear kinetics: The regression coefficient (slope) seems to be related to the type and magnitude of the non-linear kinetics. Let assume three cases of slope $b=0.5,1.0$ and 2.0. Thus;

$$
\begin{aligned}
& \mathrm{y}=\mathrm{ax}^{0.5} \ldots \ldots \ldots \text {. Type } 1 \\
& y=a x^{1} \ldots \ldots . . \text { Type } 2 \\
& y=a^{2} \cdots \cdots . . \text { Type } 3
\end{aligned}
$$

When the slope, $b$ equals nearly 1.0 (Type 2 ), it can be defined as linear kinetics. Howev$\mathrm{er}$, if $\mathrm{b}$ is definitely less than 1.0 (Type 1 ), the plasma concentration does not increase doseproportionally, and if $b$ is definitely greater than 1.0 (Type 3), the plasma concentration increases super-proportionally. Both types 1 and 3 are defined as non-linear kinetics. The type of the non-linear kinetics can be qualitatively differentiated either by the slope $b$ is less than 1.0 or greater than 1.0, and the magnitude of the non-linear kinetics can be expressed by the extent in dissociation of the slope $b$ from 1.0. A retrospective investigation of 71 toxicokinetic studies indicated that the slope $b$ ranged from nearly zero to above 2.0 and that the estimated $b$ was less than 0.7 in $33 \%$ toxicity studies and greater than 1.3 in $20 \%$ toxicity studies (Igarashi, 1994). The slope $b=1.07$ estimated from data in Table 1 indicates that the plasma concentration of the test compound in the present dog toxicity study was dose-dependent in a manner of the linear kinetics.

The following is the conclusion from the present case study. In toxicokinetics studies, sex differences of animals, change in plasma concentration during the course of repeated dose toxicity studies and the dose-concentration relationship should be examined. The analysis of variance may be the most practical and robust method for the statistical analysis of those factorial effects and their interactions. Logarithmic transformation of concentration data before the statistical analysis seems to be essential for toxicokinetic studies because of the following reasons :

1) Logarithmic transformation of concentration data makes the heterogeneous variance of data homogenous. This is especially noteworthy in toxicity studies where test doses rise over a wide range from therapeutic dose level to toxic dose level, and therefore, plasma concentration data usually vary over an extremely wide range. Therefore, the concentration data should be logtransformed before the regression analysis of dose-concentration relationship.

2) One empirically knows that untransformed data of plasma concentrations do not verify the normality because the distribution is usually deformed by existence of some extraordinarily large figures. Therefore, median values are sometimes used to represent a group of plasma concentration data instead of arithmetic mean values. However, the estimation of median values for a group of small size (say, $n=4$ ) is irrelevant. The logarithmic transformation is essential for the statistical analysis of the concentration data as a parametric data. The analysis of variance of log-transformed data makes it possible to efficiently conduct toxicokinetic studies and to get study conclusions integrated of effects of sex difference, change due to time course and dose-dependency and their interactions.

3) Analysis of dose-dependency is the most important subject in toxicokinetic study. The logarithmic transformation of data makes it possible to quantitatively evaluate the non-linear kinetics of test compounds. The regression coefficient (slope) estimated from the log-dose and log-concentration relationship indicates the type and magnitude of the non-linear kinetics.

\section{ACKNOWLEDGMENTS}

The author expresses heartfelt thanks to Emeritus Professor David J. Finney (University of Edinburgh, UK) and to Professor of Applied Statistics Tadakazu Okuno, Science University of Tokyo for their very kind suggestions on the statistical issues and a lot of valuable advice for preparing this manuscript.

\section{REFERENCES}

FDA Notice (1994) : International Conference on Harmonization; Draft guideline on the assessment of systemic exposure in toxicology studies. Federal Register, 59, 9755-9760. 
Igarashi, T. (1994) : The use and abuse of toxicokinetics : What does actual data tell researchers? Drug Inf. J., 28, 285-293.

Midha, K. K., Ormsby, E. D., Hubbard, J. W., McKay, G., Hawes, E. M., Gavalas, L., McGilveray, I. J. (1993) : Logarithmic transformation in bioequivalence: Application with two formulations of perfenazine. J. Pharmaceutical Sciences., 82, 138-144.
Westlake, W. J. (1988) : Bioavailability and bioequivalence of pharmaceutical formulations. In Biopharmaceutical Statistics for Drug Development (Peace, K. E., ed.), pp. 329-352, Marcel Dekker, Inc., New York.

WHO draft guideline on marketing authorization requirements: Interchangeable multi-source pharmaceutical products. Consultative document ; 6 December ; 1993. 